\title{
CrónICA
}

\section{Acreditación de Programas de Formación de Post-Título en Medicina}

\section{Post-Graduate Programs Accreditation IN MEDICINE}

En la era de la globalización, Chile comparte con numerosos países los problemas relacionados con la educación superior y, en particular, con la educación médica de pre y post-título. El tema de la acreditación, la certificación y la recertificación han ido recibiendo cada vez más atención en los principales foros y discusiones de educación superior, incluida la educación médica.

Como consecuencia de ello, en los últimos años se han incorporado en el país normativas legales que están modificando en forma significativa el escenario en el que se desamolla la educación supenior. Así, por ejemplo, la Ley de Aseguramiento de la Calidad de la Educación Superior (№ 20.129) promulgada en el año 2006 tuvo como consecuencia la creación de la Comisión Nacional de Acreditación (CNA). Esta CNA es un organismo público, autónomo, cuya función es verificar y promover la calidad de las Universidades, Institutos Profesionales y Centros de Formación Técnica y de las carreras y programas que ellos ofrecen. Para su mejor funcionamiento, la CNA ha decidido dividir su funcionamiento general en tres áreas principales: el área de la educación de pre-grado, la de post-grado general y una categońa especial, el área del post-título médico. De esta forma, la acreditación de los programas y centros formadores de las diversas especialidades médicas y las subespecialidades del país ha recibido un trato especial, considerando su complejidad e importancia.

La misma Ley № 20.129 prevé la formación de agencias privadas de acreditación de centros y programas de especialidades médicas, primarias y derivadas. A partir de ahora será la CNA el organismo que deberá autorizar legalmente a estas "agencias acreditadoras de centros y programas", supervisando su normal funcionamiento. En la actualidad hay a lo menos 5 agencias privadas que han solicitado su autorización legal ante la CNA, aunque se desconoce aún cuál será su rol futuro en el ámbito de la educación médica.
Preocupada por el problema de las especialidades médicas en Chile y los cambios que se avecindaban, la Academia de Medicina del Instituto de Chile invitó a mediados de 2005 a los representantes de la Asociación de Facultades de Medicina de Chile (ASOFAMECH), la Asociación de Sociedades Científicas Médicas de Chile (ASOCIMED), el Colegio Médico de Chile AG y de otras instituciones médicas del país para discutir este complejo tema. Surgió entonces un trabajo conjunto, donde estas instituciones participaron activamente en los procesos relacionados con la acreditación de centros y programas. Finalmente y luego de un arduo trabajo, en julio de 2007 se creó una Agencia Acreditadora de Programas y Centros Formadores de Especialistas Médicos (APICE) que se ciñe a las exigencias de la Ley de Aseguramiento de la Calidad de la Educación Superior. En este momento la APICE se encuentra en los trámites legales para la personalidad jurídica, y ya muy pronto deberá postular por la autorización de la CNA para poder iniciar su pleno funcionamiento como agencia acreditadora del post-título médico en Chile.

Es digno destacar que la acreditación de centros y programas de formación de especialistas en medicina la inició en Chile la Asociación de Facultades de Medicina (ASOFAMECH) en 1980 - hace casi 30 años!- y la mantuvo hasta marzo de 2008, no sólo autorizando o desautorizando los programas, sino también estableciendo los cupos de médicos en formación para cada programa. Es más, no infrecuentemente esta Asociación concedía acreditaciones condicionadas a la introducción de ciertas modificaciones, contribuyendo significativamente al perfeccionamiento de la formación de especialistas clínicos. Lo que ahora está cambiando es que el proceso de acreditación de programas está adquiriendo un respaldo legal y le será encargado a agencias autorizadas, como la ya mencionada APICE.

Otro tema vinculado a la acreditación de programas y centros formadores por tratarse también de un control de calidad en medicina, es el de la certificación de los profesionales especialistas. Los médicos chilenos iniciaron el proceso de reconocimiento de las especialidades y los diversos especialistas y subespecialistas en 1984, por iniciativa de la Sociedad 
Médica de Santiago. En esa época un grupo de dirigentes médicos de nuestro país comenzó a reunirse sistemáticamente con el objeto de dar cuerpo a un organismo autónomo que definiera a las principales especialidades médicas y certificara a los médicos especialistas de Chile. Nació así entonces la Corporación Nacional Autónoma de Certificación de Especialidades Médicas (CONACEM), entidad autónoma que ha permitido ordenar y certificar en forma significativa al complejo universo de los médicos especialistas. CONACEM ha hecho una gran labor, normando los criterios para ser reconocidos como especialistas en 50 especialidades médicas y habiendo entregado 9.627 certificaciones. La idoneidad de CONACEM es reconocida públicamente y sus certificaciones son muy valoradas para la asignación de cargos profesionales, tanto en el área pública como en la privada.

Tal como está ocumiendo con la acreditación de programas y centros de formación de especialistas, en virtud de la Ley de Autoridad Sanitaria (№ 19.937) y de su Reglamento pendiente, la precisión de cuáles son las especialidades clínicas, los requisitos para obtener la certificación y la certificación misma están obteniendo un respaldo legal. Probablemente parte de este proceso quedará también en manos de agencias certificadoras, muy similares al CONACEM. No debemos olvidar que en Chile todo este proceso de certificación voluntaria tiene casi un cuarto de siglo de experiencia y ha servido de modelo para la implementación de la certificación en otros países. En el ámbito de la certificación de los médicos, CONACEM ha ido más allá y está actualmente abocada al complejo problema de la recertificación de los especialistas, la que espera tener implementada en el presente año. El gran trabajo realizado por CONACEM en todos estos años se asegurará si el reglamento contemplado en la Ley de Autoridad Sanitaria que se encuentra en la Contraloría General de la República reconoce a una institución similar a ésta como garante mínimo de la condición de médico especialista o de especialista derivado. Las principales instituciones relacionadas con la educación de postítulo médico confían en que este reglamento va a respetar el enorme trabajo realizado por CONACEM en estas décadas.

En 1999, un proyecto de Ley proveniente de la Cámara de Diputados pretendió corregir la desigual distribución de médicos en el país, condicionando la formación de especialistas a una larga permanencia en regiones y facultando a la autoridad sanitaria a certificar especialistas en forma autónoma. Este proyecto nunca llegó a concretarse, pero movilizó tanto al sector médico como a los profesionales afines a analizar los temas de la certificación y la acreditación de programas de especialistas en forma cada vez más organizada. Una de las notables consecuencias de este proyecto fue el nacimiento de la Asociación de Sociedades Científicas Médicas de Chile (ASOCIMED) que, agrupando a 30 sociedades científicas médicas, se abocó a la defensa de la calidad de la medicina nacional comenzando por la defensa de la certificación rigurosa y técnica de los especialistas.

Es interesante caer en la cuenta que hoy, ante el crecimiento explosivo de las Escuelas y Facultades de Medicina se ha sentido la necesidad de imponer controles de calidad con respaldo legal y encargados a agencias especializadas, unas en la certificación de los profesionales especialistas y otras en la acreditación de los centros y programas formadores de especialistas. Estas agencias vienen a reemplazar lo que las instituciones médicas -Academia de Medicina del Instituto de Chile, Sociedades Médicas, Colegio Médico y Facultades de Medicina- con transparencia y probidad en su lucha por garantizar la calidad de los especialistas médicos, vienen realizando desde la década de 1980-89, sin un respaldo legal pero sí con la fuerza moral que tienen las instituciones mencionadas. Es de esperar que los niveles de exigencia se mantengan y que ésta sea una oportunidad para perfeccionar la calidad y no una para rebajar los estándares.

Todo este proceso no ha sido fácil $\mathrm{y}$ ha requerido de un particular esfuerzo de toda la comunidad médica y de especialistas médicos de Chile, que por razones de tiempo no podemos singularizar acá. Sólo resta señalar que, en las últimas décadas, las instituciones médicas del país han dado múltiples evidencias de madurez, transparencia y probidad en su lucha por garantizar la calidad de los especialistas médicos. Su afortunado encuentro en CONACEM, en 1984, para la certificación de los especialistas, y ahora en la APICE, para la acreditación de los centros y programas, ciertamente otorga garantías de un desarrollo responsable del post-grado médico en Chile.

\section{Dr. Andrés Heerlein L.}

Presidente de la Asociación de Sociedades Científicas Médicas de Chile (ASOCIMED) 


\section{Revisión por pares en la Revista Médica de Chile, AÑo 2007}

\section{Peer-reviewers in Revista Médica de Chile, YEAR 2007}

La colaboración de los revisores externos, voluntaria, desinteresada y anónima (para los autores) es uno de los pilares que sostienen la calidad de las revistas científicas y se ha hecho cada día más importante al aumentar la complejidad metodológica de los trabajos, en el área científica y en las especialidades clínicas. Esta labor debe ser conocida por los lectores y por las instituciones en que trabajan dichos revisores. Por ese motivo, una vez al año y en el número de julio de la Revista, se identifican las personas que durante el año precedente (enero a diciembre) revisaron al menos un manuscrito. Durante 2007 intervinieron 433 personas; en 2006 fueron 495; en 2005, 445 y en 2004 fueron 419. La mayoría de ellos revisaron dos o tres manuscritos en el año y quienes figuran en la nómina de Miembros del Comité Editorial Asesor de la Revista, revisaron generalmente un número aún mayor. Esta crónica es la oportunidad de agradecer su colaboración, en nombre de los editores de la Revista Médica de Chile y del Directorio de la Sociedad Médica de Santiago Sociedad Chilena de Medicina Interna. La nómina que se publica a continuación es un documento que acredita su inapreciable participación en el proceso de "revisión por pares".

$\begin{array}{ll}\text { A } & \\ \text { Abarca } & \text { V Katia } \\ \text { Acevedo } & \text { B Juan Carlos } \\ \text { Acuña } & \text { M Raúl } \\ \text { Acuña } & \text { P Mónica } \\ \text { Acuña } & \text { R Julia } \\ \text { Agar } & \text { M Ana María } \\ \text { Ahumada } & \text { O Mónica } \\ \text { Albala } & \text { B Cecilia } \\ \text { Alegría } & \text { Q Silvia } \\ \text { Alfaro } & \text { L Jorge } \\ \text { Aller } & \text { R Felipe } \\ \text { Alliende } & \text { R Angélica } \\ \text { Almagro } & \text { L Enrique } \\ \text { Alvarez } & \text { A María Isabel } \\ \text { Alvarez } & \text { Z Manuel } \\ \text { Alvo } & \text { A Miriam } \\ \text { Amat } & \text { V José } \\ \text { Anguita } & \text { C Timoleón } \\ \text { Arancibia } & \text { H Francisco } \\ \text { Araos } & \text { H Daniel } \\ \text { Araya } & \text { H Iván } \\ \text { Ardiles } & \text { A Leopoldo } \\ \text { Arellano } & \text { H Leonardo } \\ \text { Armando } & \text { R Silvia } \\ \text { Arrese } & \text { J Marco } \\ \text { Arriagada } & \text { B Paulina } \\ \text { Arteaga } & \text { H Oscar } \\ \text { Arteaga } & \text { L Antonio } \\ \text { Arteaga } & \text { U Eugenio } \\ \text { Asenjo } & \text { G René } \\ \text { Asenjo } & \text { M Sylvia } \\ \text { Atalah } & \text { S Eduardo }\end{array}$

Avendaño B Isabel

Ayala R Francisco

Aylwin H Carmen Gloria

B

Balcells M Elvira

Balmaceda $\mathrm{R}$ José $\mathrm{P}$

Bannura C Guillermo

Baró A Michel

Barría S Manuel

Barros M Manuel

Barros O Felipe

Bastías S Gabriela

Beca I Juan Pablo

Bedregal G Paula

Behn T Claus

Beltrán B Carlos

Berger F Zoltan

Berríos C Ximena

Bertín C Pablo

Biagini A Leandro

Bianchi P Marcelo

Bianchi S Víctor

Bitar H Patricia

Blamey D Rodrigo

Blanco C Rafael

Blanco R Antonieta

Blümel M Juan Enrique

Bogado S Justo

Bono M María Rosa

Borzone $\mathrm{T}$ Gisela

Brahm B Javier

Bronfman F Lucía 


\begin{tabular}{|c|c|c|c|}
\hline Brunser & $\mathrm{T}$ Oscar & Cortés & M Fanny \\
\hline Bueno & E Beatriz & Cosoi & P Eduardo \\
\hline Bunout & B Daniel & Cotera & F Alejandro \\
\hline Burrows & A Raquel & Cruz & S Adolfo \\
\hline Bustamante & Z Marco & $\begin{array}{l}\text { Csendes } \\
\text { Cuchacovich }\end{array}$ & $\begin{array}{ll}\mathrm{J} & \text { Attila } \\
\mathrm{T} & \text { Miguel }\end{array}$ \\
\hline C & & Cuevas & M Ada \\
\hline Cabello & A Hernán & & \\
\hline Cabello & C Felipe & D & \\
\hline Cabrera & C María Elena & Dabanche & $\mathrm{P}$ Jeanette \\
\hline Cáceres & C Texia & De Aretxabala & U Xabier \\
\hline Calvo & B Alfonso & De la Fuente & H María \\
\hline Calvo & A Mario & De la Maza & C María Pía \\
\hline Campero & S Mario & De la Sotta & T Raúl \\
\hline Campino & J Carmen & Delgado & D Carolina \\
\hline Campusano & M Claudia & Descouvieres & C Carlos \\
\hline Canals & L Mauricio & Díaz & A Patricia \\
\hline Canals & L Claudio & Díaz & C Jaime \\
\hline Caorsi & S Carlos & Díaz & P Orlando \\
\hline Carrasco & B Eduardo & Dighero & T Humberto \\
\hline Carrasco & N Fernando & Domenech & L Raúl \\
\hline Carvajal & C Sergio & Dörr & Z Otto \\
\hline Casar & C Carlos & Dougnac & L Alberto \\
\hline Cassorla & S Fernando & Dubernet & M Juan \\
\hline Castellón & V Enrique & Duclos & H Jaime \\
\hline Castillo & L Cecilia & Duffau & T Gastón \\
\hline Castillo & T Silvia & & \\
\hline Castro & G Pablo & $\mathbf{E}$ & \\
\hline Castro & H José & Enríquez & L Octavio \\
\hline Caviedes & S Iván & Erazo & T Ricardo \\
\hline Chamorro & B Hernán & Erazo & R Rodrigo \\
\hline Chamorro & S Adriana & Erices & O Alejandro \\
\hline Chamorro & S Gastón & Espinoza & G Ricardo \\
\hline Charlín & D Víctor & Espinoza & R Mauricio \\
\hline Chernilo & S Sara & Etérovic & M Ivo \\
\hline Chianale & B José & $\mathbf{F}$ & \\
\hline Chirdo & G Fernando & Fajuri & N Alejandro \\
\hline Chomalí & G May & Fardella & B Patricia \\
\hline Cifuentes & O Lucía & Fernández & V Alejandra \\
\hline Codoceo & R Verner & Ferreiro & P Osvaldo \\
\hline Comparini & F Beatriz & Fica & D Mauricio \\
\hline Concha & G Selim & Fierro & C Juan Alberto \\
\hline Conte & L Guillermo & Figueroa & M Claudia \\
\hline Conget & M Paulette & Figueroa & G Guillermo \\
\hline Contreras & P Jaime & Figueroa & C Gustavo \\
\hline Contreras & S Andrea & Florenzano & U Fernando \\
\hline Contreras & T Gustavo & Florenzano & U Ramón \\
\hline Cordella & M Patricia & Fuentealba & P Carlos \\
\hline Cornejo & R Rodrigo & Fuentes & G Patricio \\
\hline Corrales & V Raúl & Fullerton & U Claudio \\
\hline Cortés & G Pablo & Fuster & S Francisco \\
\hline
\end{tabular}


G

$\begin{array}{ll}\text { Gajardo } & \text { L Héctor } \\ \text { Gálvez } & \text { G Sergio } \\ \text { Gambra } & \text { A Pilar } \\ \text { García Huidobro } & \text { T Juan } \\ \text { Garrido } & \text { N Jorge } \\ \text { Garrote } & \text { A José Antonio } \\ \text { Gatica } & \text { B Arnaldo } \\ \text { Germain } & \text { A Alfredo } \\ \text { Giaconi } & \text { G Juan } \\ \text { Gil } & \text { H Lionel } \\ \text { Gil } & \text { D Rodrigo } \\ \text { Glasinovic } & \text { P Andrea } \\ \text { Godoy } & \text { F Jaime } \\ \text { Godoy } & \text { J Gonzalo } \\ \text { Goic } & \text { G Alejandro } \\ \text { Gómez } & \text { H Orietta } \\ \text { González } & \text { A Patricia } \\ \text { González } & \text { B Sergio } \\ \text { González } & \text { C Carlos } \\ \text { González } & \text { E Fernando } \\ \text { González } & \text { F Fernando } \\ \text { González } & \text { O Ximena } \\ \text { González } & \text { R Gerardo } \\ \text { González } & \text { Y Mónica } \\ \text { Grant } & \text { D Carlos } \\ \text { Grez } & \text { L Lionel } \\ \text { Guardia } & \text { B Sylvia } \\ \text { Guiraldes } & \text { C Ernesto } \\ \text { Guivernau } & \text { B Mauricio } \\ \text { Gutiérrez } & \text { C Jorge } \\ \text { Guzmán } & \text { B Sergio } \\ & \end{array}$

\section{H}

$\begin{array}{ll}\text { Harbst } & \text { S Hans } \\ \text { Harris } & \text { D Paul } \\ \text { Heerlein } & \text { L Andrés } \\ \text { Hermansen } & \text { T Carlos } \\ \text { Hernández } & \text { G Guillermo } \\ \text { Herskovic } & \text { L Jorge } \\ \text { Herskovic } & \text { L Pedro } \\ \text { Hidalgo } & \text { V Soledad } \\ \text { Hirsch } & \text { B Sandra } \\ \text { Hodgson } & \text { B María Isabel } \\ \text { Hunter } & \text { M Bessie } \\ \text { Hurtubia } & \text { N Ricardo }\end{array}$

I

$\begin{array}{ll}\text { Ibáñez } & \text { G Pilar } \\ \text { Ibáñez } & \text { C Fernando } \\ \text { Ibarra } & \text { V Humberto } \\ \text { Icaza } & \text { N Gloria }\end{array}$

Irribarra $\quad \mathrm{P}$ Verónica

Ivanovic-Zuvic R Fernando

J

Jadresic M Enrique

Jadue $\quad \mathrm{H}$ Liliana

Jalil M Jorge

Jara B Lilian

Jara G Alejandra

Jelves M Ivonne

Jensen B Cristián

Juez G Gabriela

Juliet L Chrystal

$\mathbf{K}$

Katz B Ricardo

Kauffmann Q Ronald

Koch Osvaldo

Kottow L Miguel

L

Labarca B Rodrigo

Lama T Alexis

Lango G Isabel

Larraín $\quad \mathrm{R}$ Ernesto

Larraín B Francisco

Larrañaga L Carmen

Larrondo L Milton

Latorre M Ricardo

Lavados G Pablo

Leisewitz V Thomas

Leiva $\quad \mathrm{R}$ Isabel

Liberman G Claudio

Liendo $\quad \mathrm{P}$ Federico

López K Francisco

López M José Manuel

López S Gloria

Lorca H Miriam

Lubascher C Jaime

Lutz H Alejandro

M

Macho F Pilar

Madrid S Ana María

Maggiolo G Pedro

Maiz G Alberto

Maiza R Eduardo

Majlis L Alejandro

Mallol V Javier

Margozzini M Paula

Martínez A Alejandro

Martínez C Virginia 


$\begin{array}{ll}\text { Massardo } & \text { V Loreto } \\ \text { Medina } & \text { C Eduardo } \\ \text { Medina } & \text { I Francisco } \\ \text { Melkonian } & \text { T Ernesto } \\ \text { Mella } & \text { G Iris } \\ \text { Mena } & \text { C Beltrán } \\ \text { Mezzano } & \text { A Diego } \\ \text { Mezzano } & \text { A Sergio } \\ \text { Millán } & \text { K Teresa } \\ \text { Minguell } & \text { U José } \\ \text { Miranda } & \text { C Marcelo } \\ \text { Molina } & \text { C Ramiro } \\ \text { Molinari } & \text { C Juan } \\ \text { Montenegro } & \text { A Hernán } \\ \text { Montero } & \text { L Joaquín } \\ \text { Moore } & \text { Philippa } \\ \text { Moreno } & \text { G Manuel } \\ \text { Mosso } & \text { G Lorena } \\ \text { Moyano } & \text { D Emilio } \\ \text { Moyano } & \text { S Leonor } \\ \text { Munizaga } & \text { C Fernando } \\ \text { Muñoz } & \text { C Patricia } \\ \text { Muñoz } & \text { N Sergio } \\ \text { Muzzo } & \text { B Santiago }\end{array}$

$\mathbf{N}$

$\begin{array}{ll}\text { Nagel } & \text { B Lilianette } \\ \text { Navarrete } & \text { G Claudio } \\ \text { Nazer } & \text { H Julio } \\ \text { Neira } & \text { Q Oscar } \\ \text { Norero } & \text { V Colomba } \\ \text { Noriega } & \text { R Luis Miguel }\end{array}$

$\mathbf{0}$

$\begin{array}{ll}\text { Ojeda } & \text { F José Miguel } \\ \text { Olivares } & \text { G Manuel } \\ \text { Olmos } & \text { C Alfonso } \\ \text { Orlandi } & \text { J Luis } \\ \text { Ortega } & \text { P Rinna } \\ \text { Ortiz } & \text { P Armando } \\ \text { O’Ryan } & \text { G Miguel } \\ \text { Osorio } & \text { S Guido } \\ \text { Oyarzún } & \text { G Manuel } \\ \text { Oyarzún } & \text { E Enrique } \\ \text { Oyonarte } & \text { G Miguel }\end{array}$

$\mathbf{P}$

$\begin{array}{ll}\text { Palavecino } & \text { S Ricardo } \\ \text { Palestini } & \text { Q Mario } \\ \text { Palisson } & \text { E Francisco } \\ \text { Palma } & \text { B Cristián } \\ \text { Palma } & \text { C Renato }\end{array}$

$\begin{array}{ll}\text { Paolinelli } & \text { G Paola } \\ \text { Passalacqua } & \text { R Walter } \\ \text { Peirano } & \text { C Patricio } \\ \text { Pemjean } & \text { G Alfredo } \\ \text { Pereira } & \text { G Jaime } \\ \text { Pérez } & \text { A Patricia } \\ \text { Pérez } & \text { C Carlos } \\ \text { Pérez } & \text { P Gilberto }\end{array}$

Pérez Cotapos S María Luisa

Perret P Cecilia

Pidal M Paola

Pineda B Pedro

Pino Z Paulina

Pinto $\quad C$ María Eugenia

Pinto C Juan

Plubins M Luis

Poniachik T Jaime

Prieto D Juan Carlos

Puelma P Alvaro

Puga L Bárbara

Püschel I Klaus

Q

Quera P Rodrigo

Quijada H Mario

R

Rada G Gabriel

Raggio M Ximena

Ramírez G María Cristina

Repetto L Gabriela

Reyes V José Miguel

Reynolds $\mathrm{H}$ Enrique

Ribalta S José

Riedemann G Stella

Rigotti R Attilio

Ríos S Rafael

Riquelme P Arnoldo

Roa E Iván

Roa S Juan Carlos

Rocabado S Mariano

Rodillo B Eliana

Rodrigo S Ramón

Roessler B Emilio

Rojas C María Graciela

Rollán R Antonio

Román R Rossana

Rosselot J Eduardo

Rothhammer E Francisco

Rozowski N Jaime

Rugiero P Elsa 
S

$\begin{array}{ll}\text { Sabbagh } & \text { P Eduardo } \\ \text { Sáenz } & \text { F Roque } \\ \text { Salamanca } & \text { F Lucía } \\ \text { Salas } & \text { D Cristián } \\ \text { Salazar } & \text { O Flavio } \\ \text { Saldías } & \text { P Fernando } \\ \text { Salinas } & \text { R Rodrigo } \\ \text { Salvo } & \text { C Karen } \\ \text { Santos } & \text { A Manuel } \\ \text { Santos } & \text { M José Luis } \\ \text { Sapunar } & \text { Z Jorge } \\ \text { Sazunic } & \text { Y Ivo } \\ \text { Scharager } & \text { G Judith } \\ \text { Schiattino } & \text { L Irene } \\ \text { Schilkrut } & \text { G Raúl } \\ \text { Sedano } & \text { L Manuel } \\ \text { Segovia } & \text { D Isabel } \\ \text { Segovia } & \text { R Erico } \\ \text { Seijas } & \text { B Daniel } \\ \text { Sepúlveda } & \text { C Jacqueline } \\ \text { Sepúlveda } & \text { J Rafael } \\ \text { Serani } & \text { M Alejandro } \\ \text { Sierralta } & \text { Z Armando } \\ \text { Silva } & \text { I Hernán } \\ \text { Silva } & \text { S Jorge } \\ \text { Sirhan } & \text { N Marisol } \\ \text { Soffia } & \text { S Pablo } \\ \text { Solari } & \text { I Aldo } \\ \text { Soriano } & \text { B Humberto } \\ \text { Soto } & \text { I Néstor } \\ \text { Sotomayor } & \text { S María Angélica } \\ \text { Soza } & \text { R Alejandro } \\ \text { Spencer } & \text { O Eugenio } \\ \text { Squella } & \text { B Fredy } \\ \text { Szanthó } & \text { P Gyorgy } \\ \text { T } & \\ \text { Tapia } & \text { I Patricia } \\ \text { Tchernitchin } & \text { V Andrei } \\ \text { Téllez } & \text { T Carlos } \\ \text { Thompson } & \text { M Luis } \\ \text { Toche } & \text { P Paola } \\ \text { Toro } & \text { U Cecilia } \\ \text { Trejo } & \text { M Carlos } \\ \text { Triviño } & \text { B Ximena } \\ \text { Trucco } & \text { B Marcelo } \\ \text { Trujillo } & \text { V Sergio } \\ \text { Turner } & \text { G Eduardo } \\ \text { U } & \\ \text { Uribe } & \\ & \\ & \\ \text { Sadro }\end{array}$

Uribe

M Mario

V

Vacarezza Y Ricando

Valderrama L Rodrigo

Valdés

S Gloria

Valdés

L Verónica

Valdivia

C Gonzalo

Valdivieso

D Andrés

Valdivieso

D Vicente

Valdivieso

Valenzuela

F Sergio

Valenzuela

A Eduardo

Valenzuela

E Jorge

$P$ Sergio

Valenzuela

Y Carlos

Vargas

Vargas

Vásquez

Vásquez

Vega

Velarde

Velasco

Véliz

Venegas

Vera

C Nelson

M Sergio

M Alicia

T Patricia

B Luis

A Victoria

F Nicolás

L Jesús

P Patricio

Verdaguer

Vergara

C Aldo

$\mathrm{T}$ Juan

Vicente

E Fernando

Vidal

P Benjamín

Villagra

Villagra

A Roberto

C Eliecer

C Roque

Villaseca

H Miguel

Von Plessing

Vukasovic

$R$ Carlos

W

Wainstein G Eduardo

Weitz V Juan Carlos

Wistuba O Iván

Wohllk G Nelson

Wolff R Marcelo

$\mathbf{Y}$

Yáñez L Alfredo

Yáñez S Paulina

$\mathbf{Z}$

Zalaquett S Ricardo

Zamorano G Jaime

Zamudio A Eduardo

Zárate B Víctor

Zúñiga D Alvaro 


\section{Publicaciones y Multimedia \\ Publications and Muttimedia}

NuEva GUÍA SOBRE LOS RIESGOS DE LA CENIZA VOLCÁNICA

En emergencias volcánicas dos elementos que generan una importante alarma son la ceniza y los gases volcánicos. La "International Volcanic Health Hazard Network (IVHHN)" ha publicado guías técnicas sobre los riesgos de la ceniza y los gases, diseñados con un contenido muy práctico, para uso y distribución masiva en casos de erupciones. "Los daños a la salud por cenizas volcánicas. Una guía para la población", brinda recomendaciones para los problemas respiratorios, oculares o cutáneos, además de recomendaciones generales para protegerse de la ceniza, o para proteger a los niños. Otro documento, "Guía de preparación antes, durante y después de una lluvia de cenizas", ofrece recomendaciones generales de preparativos a los administradores de los desastres.

Los miembros de la IVHHN, 31 en total, trabajan en diversas disciplinas científicas como vulcanología, epidemiología, toxicología y salud pública con el objetivo de determinar los efectos en la salud de las emisiones volcánicas. Las guías están disponibles en español, inglés, italiano, francés y portugués en www.ivhhn.org (ver la sección de "guidelines and databases").

\section{Página WeB PARA POBLACIONES ESPECIALES}

La Biblioteca Nacional de Medicina de Estados Unidos de Norteamérica (NLM, por sus siglas en inglés) lanzó una página web para poblaciones especiales sobre preparativos ante emengencias y desastres. El sitio contiene información sobre medidas de seguridad, evacuación, preparación, etc., para personas con discapacidades, de edad avanzada, con deficiencias auditivas y visuales, mujeres, niños, diabéticos, indígenas, extranjeros, entre otros. Adicionalmente, recoge lecciones aprendidas de desastres provocados por los huracanes Katrina y Rita y los ataques al World Trade Center, leyes y regulaciones y una guía de preparativos para el gobierno y organizaciones. La página está disponible en http://sis.nlm.nih.gov/ outreach/specialpopulationsanddisasters.html.

(Textos reproducidos de "Publicaciones y Multimedia" No 109, marzo 2008). 